\title{
An On-line Detector and Inversion Algorithm of Suspended Particles
}

\author{
Deli Jia $^{\mathrm{a}, *}$, Tong Guo ${ }^{\mathrm{a}}$, Zhenkun Zhu ${ }^{\mathrm{b}}$, Quanbin Wang ${ }^{\mathrm{a}}$, Yan Wang ${ }^{\mathrm{a}}$, and Yanping Wang \\ ${ }^{a}$ PetroChina Research Institute of Petroleum Exploration \& Development, PetroChina Company Limited, Beijing, 100000, China \\ ${ }^{b}$ Daqing Research Institute of Oil Production Engineering, Daqing Oil \& Gas Company Limited, Daqing, 163000, China \\ ${ }^{c}$ Xinli Oil Production Plant of Jilin Oilfield, Daqing Oil \& Gas Company Limited, Songyuan,138000, China
}

\begin{abstract}
Stratified waterflooding is the main technology for oilfield development. Continuously improving waterflooding control and utilization through fine waterflooding is a never-ending pursuit. Reinjecting the produced liquid into the formation after electrical dehydration is the main technical means for the waterflooding development of oilfields. Whether the suspended particle in the reinjection water meets the standards will directly affect the waterflooding development effect. This paper proposes and develops an on-line suspended particle detector and its inversion algorithm. In order to meet the downhole detection requirements of subsurface suspended particles in reinjection water, a laser on-line detector for suspended particle was developed based on the light scattering method, and an array structured lightinduced ring detector was designed to realize the identification of scattered light. In order to meet the engineering requirements of on-line inspection, the genetic algorithm and least-squares algorithm are used to optimize the granularity inversion calculation. The detection error and response time of these algorithms are compared and analyzed. With an error below the allowed value of $4 \%$ and a response time of $0.22 \mathrm{~s}$, the least square algorithm has more engineering application value. In order to prevent the occurrence of negative numbers after iteration of the least squares algorithm, a non-negative least square granularity inversion algorithm was designed in the practical engineering application. Based on the actual engineering data and the simulation structure, it can be concluded that the simulation values are highly consistent with the theoretical values, which proves that the laser suspended particle on-line detector and its inversion algorithm are applicable to the on-line detection system of suspended particles in the reinjection water of oilfields.
\end{abstract}

Keywords: stratified waterflood; suspended particles; laser; inversion algorithm; least square method; genetic algorithm

(Submitted on March 5, 2018; Revised on April 16, 2018; Accepted on May 21, 2018)

(C) 2018 Totem Publisher, Inc. All rights reserved.

\section{Introduction}

With the continuous development of oil production technology, the stage of primary, secondary and tertiary oil recovery came successively. Primary oil recovery is driven by natural energy, secondary oil recovery uses artificial water injection to maintain formation pressure [8,17], and tertiary oil recovery increases the oil recovery rate by changing the characteristics of the injected water. At present, secondary and tertiary oil recovery are mainly carried out, and secondary oil recovery is the main development technology $[9,10,14]$, which means that most of the oilfields are mainly developed by waterflooding. Constantly increasing waterflooding control degree and producing degree through fine water injection is a never-ending pursuit $[4,11]$.

During the waterflooding development, the water-containing crude oil produced from the underground is converted into "produced fluid" and electrically dehydrated. The separated water is called "oilfield produced water", also called "oilfield sewage". In the production of oil fields, most of the produced water from the oil fields is treated and injected back into the formation, and only a small part of the produced water is discharged outside. The produced water is treated and injected back into the reservoir to supplement the pressure of the formation, which not only enables us to recover the crude oil in water and realize the recycling of water and avoid environmental pollution, but also provides sufficient water for water injection and saves a large amount of fresh water resources. This measure has brought about remarkable economic and social benefits in the development of China's oil fields.

\footnotetext{
* Corresponding author.

E-mail address: jiadeli422@ petrochina.com.cn
} 
In this process, the quality of reinjection water is an important indicator of oilfield waterflood and an important factor affecting the waterflooding effect and cost. The quality of the injected water directly affects the waterflooding development effect of the oilfield. When the suspended solids in the oilfield reinjection water do not meet the standard, it will block the core of the formation, resulting in a decrease in the permeability of the formation. The formation permeability is an important factor affecting the oil recovery. A smaller permeability means a greater difficulty of water injection and a lower oil recovery $[19,22]$. The main factor affecting the decrease of formation permeability is the diameter and content of suspended solids in the reinjection water [1,3].

The water quality analysis of traditional injected water is mostly carried out in the way of off-line inspection, which means that on-site sampling, storage, laboratory testing, data recording and feedback to the site are performed successively. The analysis cycle of the entire process is long. If the water quality is not properly maintained, it will cause great errors in the test data, thus losing the function of supervision and guidance on production. Therefore, in order to increase oil recovery and reduce production costs, it is necessary to conduct online monitoring on the diameter and content of suspended solids in the oilfield reinjection water, so as to promptly find the over-standard water injection, and report and solve problems.

According to different measurement requirements, there are many types of particle size measurement instruments, and there are many corresponding particle measurement methods. According to its basic working principle, it can be divided into direct and indirect methods [5,16,24]. In the direct method, measurement is based on the geometry of the particles, such as sieving method and microscope method. According to a certain physical rule, a certain physical quantity of a particle under the influence of certain factors is measured and then converted into the diameter of a sphere with the same values of the same physical quantity, which is used to represent the size of the particles. This method is known as the indirect method, such as sedimentation method, electric induction method (Coulter method) and light scattering method [2,20]. Each of them has its own characteristics and application range, but most of the methods do not support online detection. The development of particle size analyzers started earlier in foreign countries. The mature foreign companies include Malvern in the United Kingdom, Coulter in the United States, Cilas in France, Sympatec in Germany, and HORIBA in Japan.

The development of China's laser particle size analyzer began in the late 1980s; some progress has been made in recent years. The influential brands include Zhuhai "OMEC", and the brands with high technical content include Jinan "Winner", Chengdu "Jingxin" and Dandong "Bettersize". However, there are still some gaps between domestic and foreign products in terms of key performance indicators such as measurement range, measurement repeatability, granularity resolution and reliability. In addition to the backward processing technology, the imperfection of the inversion algorithm also plays a great restrictive role.

In view of this, a study of on-line inspection of suspended particle in the water during zonal injection in the field of oil extraction engineering was carried out. A laser particle size analyzer was proposed and developed using light scattering method. The comparison and verification of different inversion algorithms was focused on, and engineering techniques suitable for field applications were determined.

\section{Design of suspended particle online detector}

Light scattering is currently the most widely used particle measurement method. The laser particle size analyzer is a typical instrument based on the principle of light scattering. It measures the particle size distribution of powder samples by using properties such as the amplitude, phase and polarization of scattered light related with the size and refractive index of the scattered light after the light is scattered; it uses laser as the light source and uses data processing software based on Mie scattering theory to analyze the test data.

The suspended particle on-line detector developed in this paper is shown in Fig. 1. The entire instrument includes laser, laser filter-collimated beam expander system, sample cell, Fourier transform lens system, detector and data processing system. Its core component is a combined multi-element silicon photodetector.

The working principle of the suspended particle on-line detector is as follows. The monochromatic laser beam emitted by the semiconductor laser will converge on the pinhole after passing through the microscope objective. The pinhole will filter out all the high-order scattered light and allow only the low-frequency laser to pass through. The laser beam then becomes a diverging light beam that passes through a collimating lens to form a parallel monochromatic light beam with a diameter of approximately $8 \mathrm{~mm}$. When there are no particles in the sample cell, the parallel beam passes through the Fourier lens and is focused on the center of the ring photodetector and passes through the center hole to the center detector. When there is a particle sample in the sample cell, part of the converged light beam will be scattered by particles to the detection unit and the large-angle detector of the ring detector. The intensity distribution of the scattered light obeys Mie 
theory. At this point, the Fourier lens behind the sample cell is the receiving lens (whose focal length is known), and a far field scattering pattern of scattered light is formed on the back focal plane of the lens. Particle size and particle distribution and even particle shape in the airspace are one-to-one correspondence, which satisfies the Fourier transform relationship. In addition, according to the optical theory, in the case of paraxial (i.e., small-angle scattering), scattered light with the same scattering angle of the inverse Fourier transform converges on the detector in the focal plane. The result is the same as the classic Fourier transform except that the focal length corresponds to the distance from the sample location to the detector. However, as the scattering angle increases, the focusing situation becomes worse and worse. At this time, when calculating the theoretical light energy distribution, it must be corrected; otherwise, it will cause errors. The so-called reverse Fourier transform is an optical structure in which a sample to be measured is placed behind a Fourier lens and is irradiated with converging light.

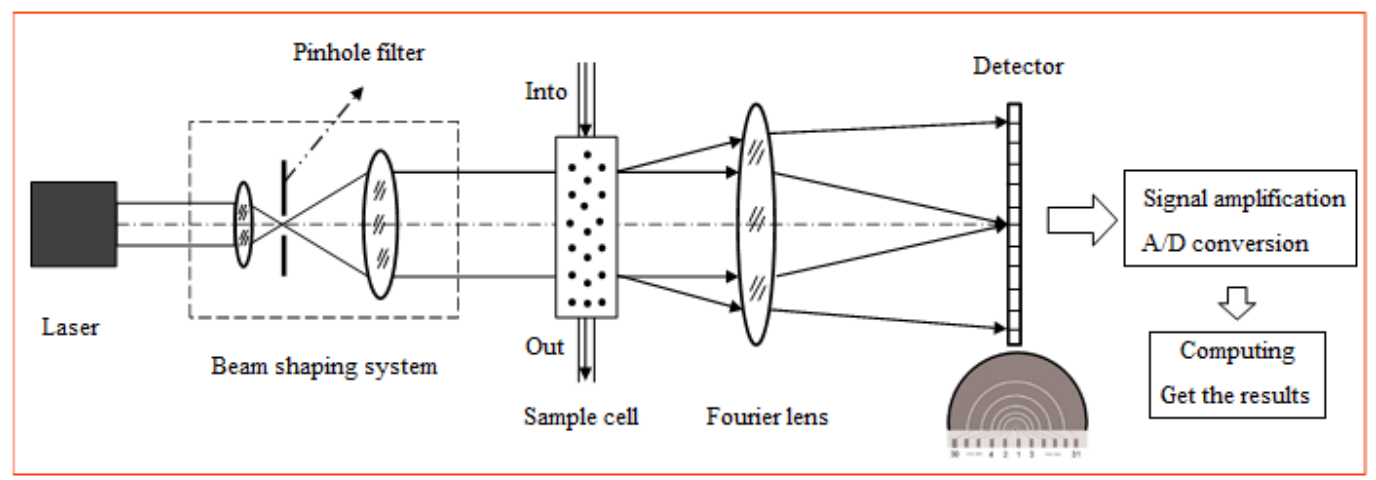

Figure 1. Structure principle of the suspended particle on-line detector

A multi-ring photodetector array is then placed on the focal plane of the receiving lens to receive the scattered light energy, and to convert it into the electrical signal to output. The central aperture (central detector) on the detector can be used to determine the allowable sample volume concentration. After amplification and A/D sampling of the received electrical signal, the particle size analysis software in the computer was used to obtain the particle size distribution.

The detector is the core component of the system and affects the performance of the test data. Because the scattered light from the particles is weak, the detector array adopts a semicircular structure to increase the detection area. $A$ small hole is opened in the center of the detector array to pass the zero-order diffracted light. The structure of the detector array is composed of concentric half rings with different radii on one silicon wafer and independent of each other. Adjacent two rings are insulated from each other. According to the optical system parameters, when the laser illumination wavelength $\lambda$ is $650 \mathrm{~nm}$ and the Fourier transform lens focal length $f$ is $50 \mathrm{~mm}$, the design of the detection ring needs to meet the requirements for particle size $(1-100 \mu \mathrm{m})$. It was calculated that the ring detector has the inner and external radius of $0.14-14$ $\mathrm{mm}$ range and an interval of $24-28 \mu \mathrm{m}$. In general, the radius of the ring detector should be slightly larger than the maximum outside diameter.

The size of the innermost ring of the detector array determines the upper limit of the measurement range, which in turn depends on the size of the center hole of the detector array. The beam emitted by the laser becomes parallel light after being expanded and collimated. The diameter of the parallel beam depends on the clear aperture of the iris diaphragm. After the parallel beam passes through the Fourier transform lens, it forms an Airy spot on its focal plane. The calculation formula for the diameter of Airy spots is expressed as follows:

$$
D_{\text {airy }}=2 \times \frac{1.22 \lambda f}{D}
$$

Where $f$ is the focal length of the Fourier imaging lens, $D$ is the clear aperture of the iris diaphragm, and $\lambda$ is the laser wavelength.

Airy spot has strong energy. If it does not pass through the detector array, it will be reflected on the surface of the detector array to form a strong reflected light, and form a strong background light after multiple reflections. The background light can coincide with part of the light scattered by the particles, which seriously affects the accuracy of the measurement results. Therefore, the center of the detector array must have large holes to allow this part of the energy to pass through. It is known that the Airy spot diameter obtained based on the design parameters is $10 \mu \mathrm{m}$, which meets the requirements. Table 1 shows the specific parameters of the detector array ring. The center detector is placed behind the center hole of the detector 
array. The center detector is a one-cell silicon detector. It is required to have a large saturation current and be able to receive the large light energy of Airy spot. A low-noise silicon detector should be selected.

\begin{tabular}{|c|c|c|c|c|c|c|c|c|c|c|c|}
\hline $\begin{array}{l}\text { Number } \\
\text { of rings }\end{array}$ & $\begin{array}{c}\text { Inner } \\
\text { radius } \\
(\mathrm{mm})\end{array}$ & $\begin{array}{c}\text { Outer } \\
\text { radius } \\
(\mathrm{mm})\end{array}$ & $\begin{array}{c}\text { Area } \\
\left(\mathrm{mm}^{2}\right)\end{array}$ & $\begin{array}{l}\text { Number } \\
\text { of rings }\end{array}$ & $\begin{array}{c}\text { Inner } \\
\text { radius } \\
(\mathrm{mm})\end{array}$ & $\begin{array}{c}\text { Outer } \\
\text { radius } \\
(\mathrm{mm})\end{array}$ & $\begin{array}{c}\text { Area } \\
\left(\mathrm{mm}^{2}\right)\end{array}$ & $\begin{array}{l}\text { Number } \\
\text { of rings }\end{array}$ & $\begin{array}{c}\text { Inner } \\
\text { radius } \\
(\mathrm{mm})\end{array}$ & $\begin{array}{c}\text { Outer } \\
\text { radius } \\
(\mathrm{mm})\end{array}$ & $\begin{array}{c}\text { Area } \\
\left(\mathrm{mm}^{2}\right)\end{array}$ \\
\hline 1 & - & 0.1300 & 0.0265 & 12 & 0.7234 & 0.8261 & 0.2500 & 23 & 3.3500 & 3.8257 & 5.3618 \\
\hline 2 & 0.1400 & 0.1598 & 0.0093 & 13 & 0.8361 & 0.9549 & 0.3340 & 24 & 3.8357 & 4.3803 & 7.0294 \\
\hline 3 & 0.1698 & 0.1940 & 0.0137 & 14 & 0.9649 & 1.1019 & 0.4448 & 25 & 4.3903 & 5.0138 & 9.2093 \\
\hline 4 & 0.2040 & 0.2329 & 0.0198 & 15 & 1.1119 & 1.2698 & 0.5907 & 26 & 5.0238 & 5.7371 & 12.0585 \\
\hline 5 & 0.2429 & 0.2774 & 0.0282 & 16 & 1.2798 & 1.4615 & 0.7825 & 27 & 5.7471 & 6.5632 & 15.7811 \\
\hline 6 & 0.2874 & 0.3282 & 0.0394 & 17 & 1.4715 & 1.6805 & 1.0346 & 28 & 6.5732 & 7.5066 & 20.6439 \\
\hline 7 & 0.3382 & 0.3863 & 0.0546 & 18 & 1.6905 & 1.9305 & 1.3654 & 29 & 7.5166 & 8.5840 & 26.9949 \\
\hline 8 & 0.3963 & 0.4526 & 0.0750 & 19 & 1.9405 & 2.2161 & 1.7992 & 30 & 8.5940 & 9.8144 & 35.2878 \\
\hline 9 & 0.4626 & 0.5283 & 0.1022 & 20 & 2.2261 & 2.5422 & 2.3677 & 31 & 9.8244 & 11.2194 & 46.1150 \\
\hline 10 & 0.5383 & 0.6147 & 0.1384 & 21 & 2.5522 & 2.9146 & 3.1123 & 32 & 11.2294 & 12.8240 & 60.2487 \\
\hline 11 & 0.6247 & 0.7134 & 0.1864 & 22 & 2.9246 & 3.3400 & 4.0868 & 33 & 12.8340 & 14.6565 & 78.6968 \\
\hline
\end{tabular}

\section{Research on Data Inversion Algorithm}

As the corresponding information about the incident light wavelength and the scattered light is known, how to obtain the information of the scattered particles or particle groups participating in the scattering is an inversion problem. There are two forms of inversion. The most common is that the system and output are known and the input needs to be figured out. Another one is that the input and output are known and the unknown system needs to be figured out. The case of measuring particle size and particle size distribution using the laser particle size analyzer belongs to the prior one. Positive problem often gives a lot of constraints. The various constraints put forward based on the actual conditions are sufficient to make the derivation provide the only stable solution. The inversion problem often does not provide a unique and stable solution because of the effects of some unknown factors, suggesting that if there is no known restriction to limit the uniqueness of the solution. This problem is "ill-posed".

According to the Mie scattering theory, the relationship between the particle size distribution of the particle or particle swarm and the scattered light intensity can be expressed as $E=T W$, and if $T$ is in full rank, the size distribution column vector $W=T^{l} E$. However, a large number of calculations have shown that the inverse problem of inversing the grain size information of particles by scattered light from particles is "ill-posed". The particle size distribution obtained according to the above formula often produces a non-positive solution, which does not conform to the actual situation, so this method is generally not adopted.

The commonly used granularity inversion algorithms can be roughly divided into two major categories, namely the distribution function definition method (also known as the non-independent model algorithm) and the free distribution method (also known as the independent model algorithm). In the non-independent model algorithm, the granularity information of the measured particle needs to be known. Generally, it is necessary to know about that the particle size distribution of the particle swarm satisfies a certain function distribution. In the independent model algorithm, it is not necessary to presuppose the particle size distribution model, but to directly obtain the particle size distribution of the particle swarm from the light energy distribution values of the actual amount of speculation. This method enables us to handle any distributed particle size distribution without having to know in advance the distribution of the measured particles. In this paper, a comparative analysis was conducted based on the actual needs of the project.

\subsection{Basic physical model analysis}

According to the Mie scattering theory, the scattering light intensity of a single spherical medium particle irradiated by a monochromatic plane wave with a wavelength of $\lambda$ and a light intensity of $I_{0}$ is expressed by:

$$
I=\frac{\lambda^{2} I_{0}}{4 \pi^{2} r^{2}}\left(i_{1}+i_{2}\right)
$$

Where $i_{1}$ and $i_{2}$ are the vertical and horizontal components of the scattered light intensity, respectively.

$$
i_{1}(a, m, \theta)=\left|\sum_{n=1}^{\infty} \frac{2 n+1}{n(n+1)}\left(a_{n} \pi_{n}+b_{n} \tau_{n}\right)\right|^{2}
$$




$$
i_{2}(a, m, \theta)=\left|\sum_{n=1}^{\infty} \frac{2 n+1}{n(n+1)}\left(a_{n} \tau_{n}+b_{n} \pi_{n}\right)\right|^{2}
$$

Where $n$ is a positive integer and $m$ is the complex refractive index of particles; $a_{n}$ and $b_{n}$ are the complex amplitude coefficients of the electric vector and magnetic vector of the $n^{\text {th }}$ wavelet and are determined by the Bessel function; $\pi_{n}$ and $\tau_{n}$ are determined by the Legendre function and its derivative and are related to the scattering angle $\theta$ of the scattered light.

Using equations (2), (3) and (4), the scattered light intensity distribution of a single particle sphere irradiated by a plane wave is simulated, as shown in Fig. 2. It can be seen that there are a series of extremely large or small concentric ring peaks in the scattered light intensity distribution in the spatial region, which shows the importance of the ring detector for the particle size detector.

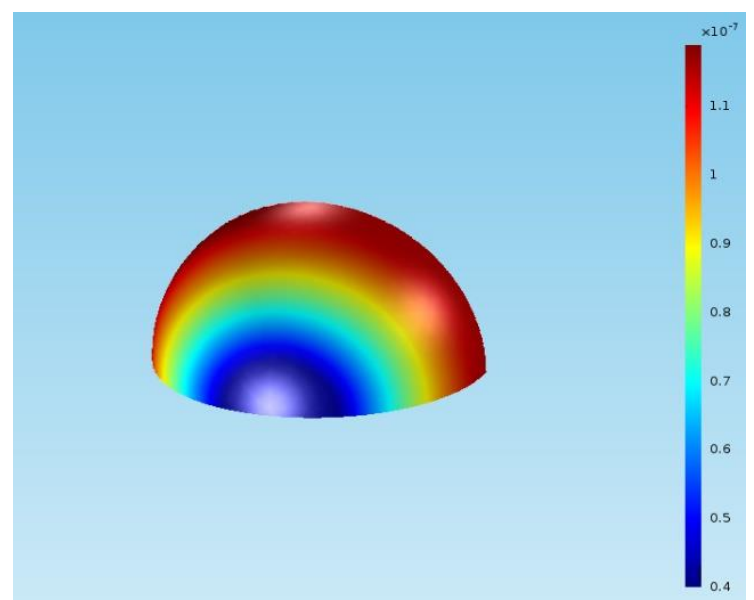

Figure 2. Schematic diagram of single-particle scattered light

The scattered light intensity is related to the particle size, refractive index, and scattering angle. The large particle has a small scattering angle and the small particle has a large scattering angle. The calculation results are shown in Fig. 3.

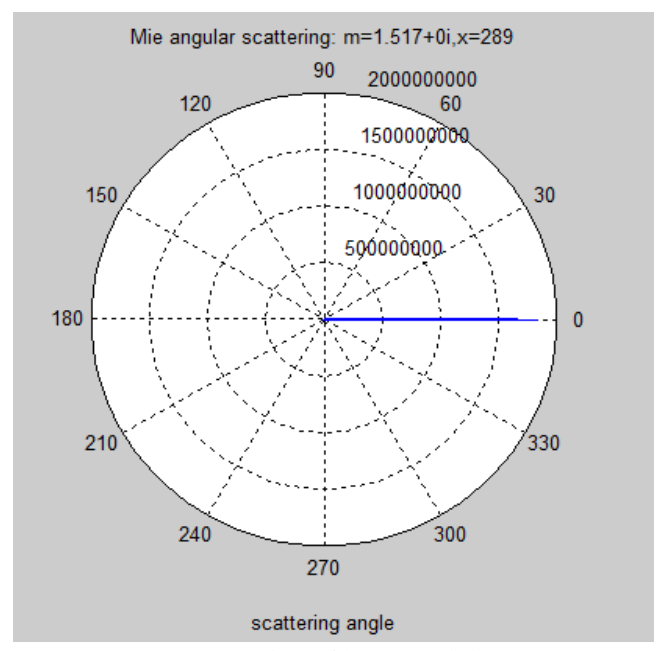

a. Scattering of large particle

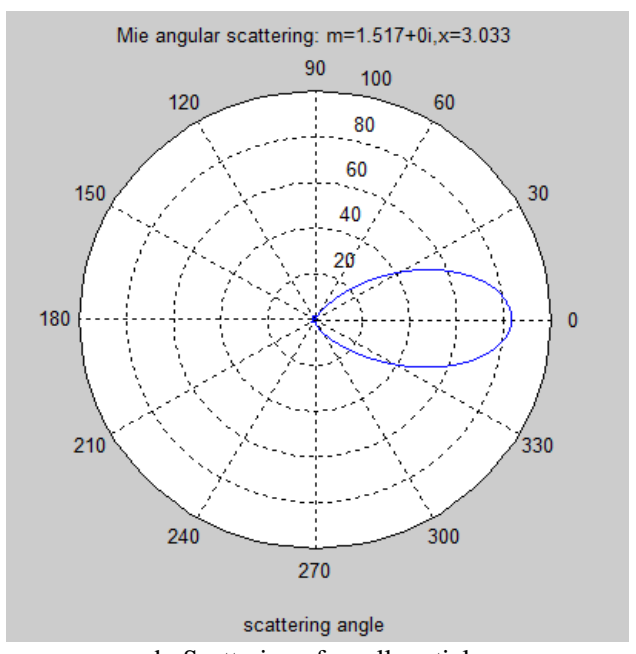

b. Scattering of small particle

Figure 3. Relationship between scattering intensity and scattering angle for different particle size

According to the spatial distribution of scattered intensity of single particles, without considering the multi-particle system under complex scattering, the scattered intensity $E_{N}$ is the incoherent superposition of the light intensity of multiple particles in different spaces. The expression is as follows:

$$
E_{N}=\sum_{i} \frac{W_{i}}{D_{i}^{3}} \int_{\theta_{n}}^{\theta_{n+1}}\left(i_{1}+i_{2}\right) \sin \theta d \theta
$$


Where $i$ is the number of particle size category and $n$ is the number of rings; $W_{i}$ is the particle size distribution; $\theta_{n+l}$ and $\Theta_{n}$ are the scattering angles of the inner and outer diameters, respectively.

Formula (5) after discretization can be written into a matrix: $E=A W$

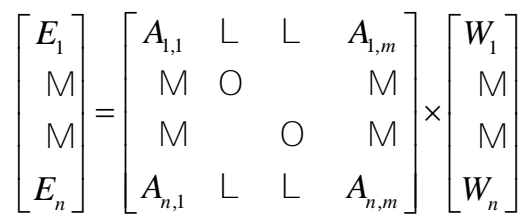

Where $\left(E_{l} \ldots \ldots E_{n}\right)$ is the light energy collected by the $j^{\text {th }}$ ring of the detector (i.e., the output energy value of the detector in the experiment); $\left(W_{1} \ldots \ldots W_{n}\right)$ is the percentage of particles in the $i^{t h}$ particle size interval (i.e., the result of the experiment); $A_{i j}$ is a light energy distribution coefficient matrix, and its physical meaning is the energy of light falling on the photodetector rings generated by the particle swarm with the particle size in the $i^{\text {th }}$ particle size interval.

\subsection{Calculation of light distribution coefficient matrix A}

The overall algorithm of the particle size analyzer is performed around the solution of matrix formula (6). In order to solve the particle size distribution $W$ of each interval, the calculation of the light energy coefficient matrix $A$ is particularly important. The solution process of the light energy coefficient matrix $A$ belongs to the first kind of Fredholm integral equation. From equations (2) to (5), it can be seen that the light energy coefficient matrix is related to the detector design parameters and the refractive index of the measuring material, which means that different detector design parameters correspond to different light energy coefficient matrix $A$. According to the design parameters of the detector (as shown in Table 1), the normalized light energy matrix $A$ is calculated, as shown in Fig. 4.

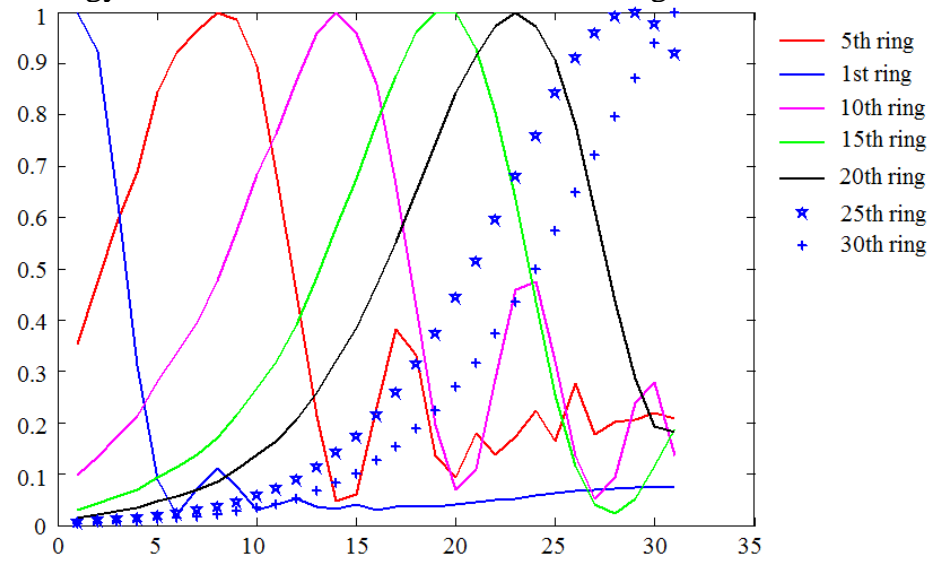

Figure 4. Light energy coefficient matrix $A$ of the 1 st ring, 5th ring, 10th ring, 15th ring, 20th ring, 25th ring and 30th ring.

When the light energy coefficient matrix $A$ is known, the particle size distribution $W$ can be obtained by performing the inversion calculation using the formula (6).

\subsection{Inverse algorithm design and comparative analysis}

According to the distribution of the actual particles, the particle size distribution inversion algorithm is roughly divided into non-independent model algorithms and independent model algorithm.

Non-independent model algorithm (distribution function definition method): Presuppose that the particle population satisfies a specific function, and continuously adjust the parameters to find a set of parameters (usually no more than 6 parameters) that make the light energy target residual smallest when the particle size distribution of the uniquely determined particle swarm on the detector is compared with the measured light energy distribution [12,15,23]. In order to avoid the influence of initial value selection on inversion results, the use of genetic algorithm is common in the non-independent model algorithm [7,21,25]. Independent model algorithm: The particle size distribution of the granular system is obtained by solving the first kind of Fredholm integral equation from the actually detected light energy distribution value. Theoretically, any particle size distribution curve can be obtained. Most of the independent model algorithms are based on 
the principle of least squares $[6,18]$.

Regardless of the inversion algorithm used, in the calculation process of the laser particle size analyzer, the scattered light intensity value $E$ collected by the ring detector is compared with the theoretical calculation value $E 0$, and the optimization method is selected to best fit the calculated value with the actual measurement value, thereby inversely deriving the particle size distribution $W$ [13]. The above-mentioned two inversion algorithms (genetic algorithm and least squares algorithm) are studied below.

a) When the genetic algorithm is used for inverse operation, the theoretical particle size distribution is set first and the Rosin-Rammler function is obeyed (as shown in Fig. 5) to get the formula (7):

$$
f_{R-R}(\mathrm{D})=\frac{k}{\bar{D}}\left[\frac{D}{\bar{D}}\right]^{k-1} \times \exp \left[-\frac{D}{\bar{D}}\right]^{k}
$$

Where $K=4, \bar{D}=4.5 \times 10^{-5}$

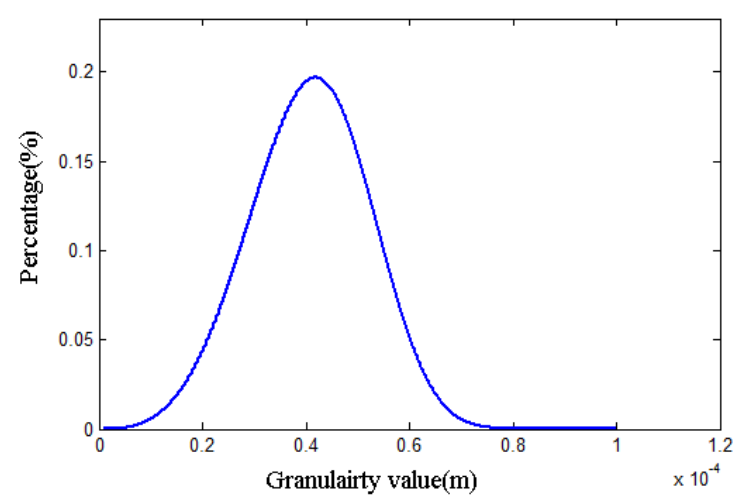

Figure 5. Theoretical particle size distribution

The inversion operation is based on the genetic algorithm. Each cycle of the calculation process undergoes fitness calculation, proportional selection operation, single-point crossover operation, and mutation operation. The basic calculation flow of the genetic algorithm is shown in Fig. 6. Comparing the simulation results of genetic algorithm with those of theoretical distribution, the conclusion is shown in Fig. 7. The error of particle size distribution simulation results is within $1 \%$, and the program running time is $8.35 \mathrm{~s}$. It can be seen that the precision of genetic algorithm is high, but the operation time is long.

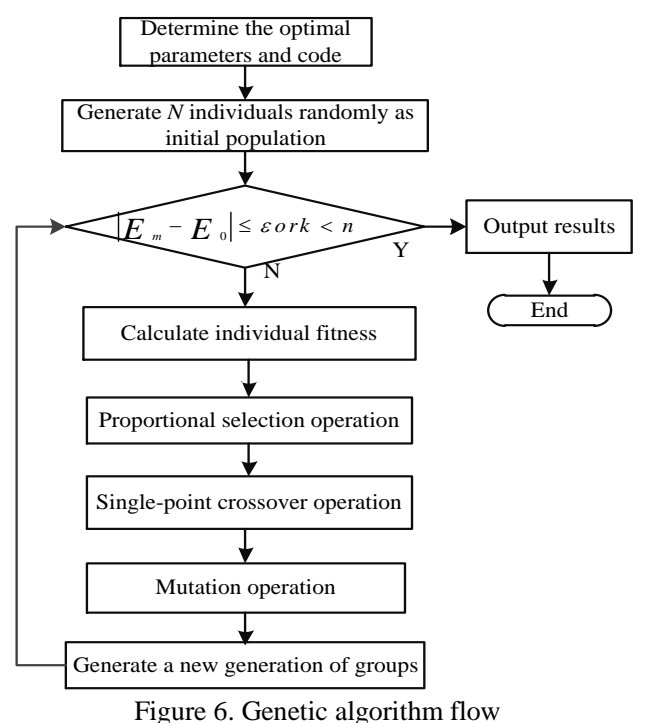




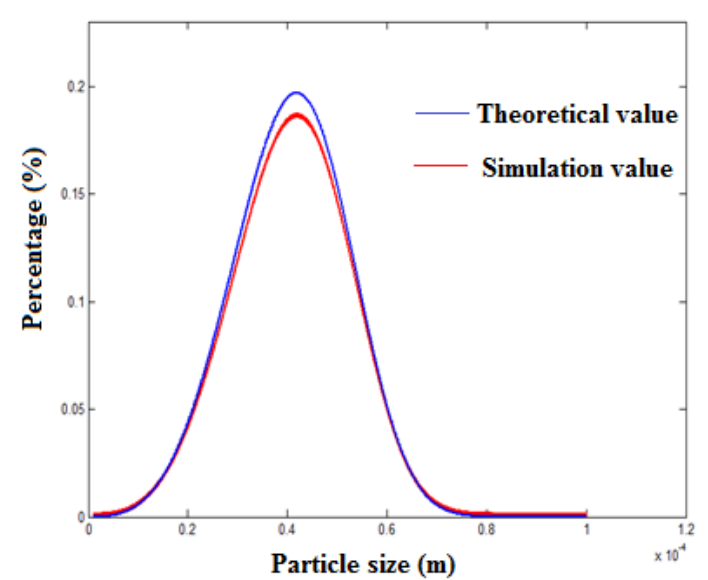

Figure 7. Comparison between theoretical and simulated values of genetic algorithm

b) When the least squares algorithm is used for inversion operation, the basic calculation flow is shown in Fig. 8 . The simulated particle size distribution based on the least squares algorithm is compared with the theoretical distribution. As shown in Fig. 9, the error of the particle size distribution simulation results is within $4 \%$, and the program running time is $0.22 \mathrm{~s}$.

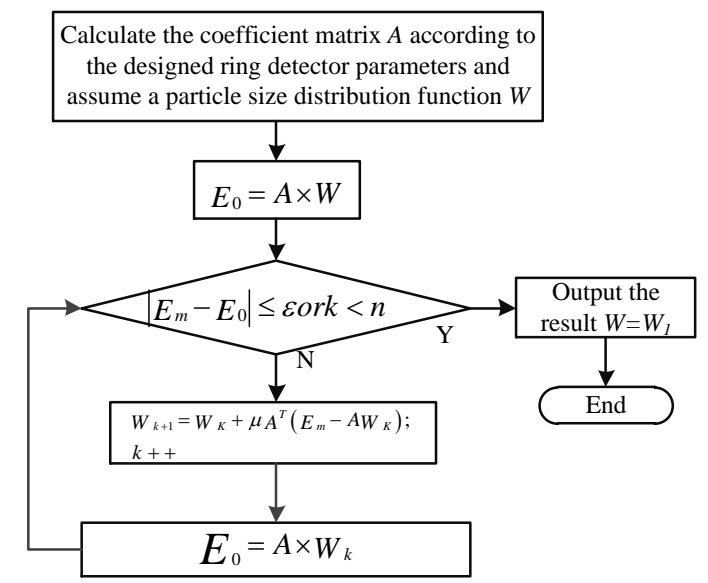

Figure 8. Least-squares algorithm flow

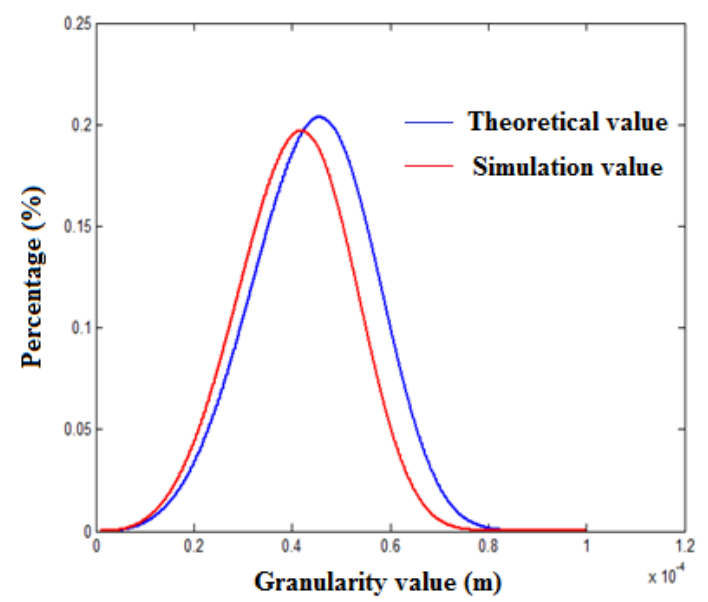

Figure 9. Comparison between theoretical and simulated values of least squares algorithm

In summary, the genetic algorithm has higher calculation accuracy than the least squares algorithm, but its calculation time is too long. If the genetic algorithm is used for inversion calculation, real-time online detection requirements cannot be met. 


\section{Engineering design and analysis of least squares inversion algorithm}

The on-line detection system of suspended particles in oilfield reinjection water adopts the least squares algorithm. At the same time, in order to prevent the negative result (inconsistent with the actual situation) after the iteration, it is still necessary to add non-negative constraints in the actual calculation; that is, there is a need to design targeted non-negative least squares (NNLS).

For the problem $f=E x$, the main purpose of non-negative least squares is $\min \|E x-f\|^{2}$ to ensure that $x \geq 0$. The nonnegative Least Squares core flow designed in this paper is as follows:

a) Set $P=\phi, \mathrm{L}=\{1,2, \quad, \mathrm{n}\}, x=0$

b) Calculate the $n$-dimensional array $w=E^{T}(f-E x)$

c) If $L=\phi$ or $w_{j} \leq 0(j \in L)$, perform steps $\left.a\right)$ and $b$ )

d) Find the subscript $t \in L$ of $w_{t}=\max \left\{w_{j} \mid j \in L\right\}$

e) Move $t$ from array $L$ to array $P$

f) Define matrix EP

column $j$ of $E_{P}= \begin{cases}\text { column } j \text { of } E & \text { (if } j \in P \text { ) } \\ 0 & \text { (if } j \in L \text { ) }\end{cases}$

Calculate $E_{P} z=f$ to figure out $z$. If $j \in L$, zj is 0

g) If all subscripts $j$ in the $P$ array have $z_{j}>0$, then set $x=z$ and execute program step a)

h) Otherwise find parameter $q \in P$, so that $\frac{x_{q}}{x_{q}-z_{q}}=\min \left\{\frac{x_{j}}{x_{j}-z_{j}} \mid z_{j} \leq 0, j \in P\right\}$

i) Set $\alpha=\frac{x_{q}}{x_{q}-z_{q}}$

j) Iterate $x=x+\alpha(x-z)$

k) Move all subscripts of $x_{j}=0(j \in P)$ from array $P$ to array L. Perform step f)

l) End the program

According to the above main program calculation steps, the actual designed laser particle size analyzer system parameters are used for design and Matlab software is used for non-negative least squares simulation. The results are shown in Fig. 10. The simulated values are highly consistent with the theoretical values, which proves that it is feasible to use nonnegative least-squares method for the on-line detection system of suspended particles in the oilfield reinjection water.

\section{Conclusions}

Stratified waterflooding is the main technology for China's oilfield development. The stratified waterflooding technology has gone through more than 60 years of development, and has played an important role in sustaining high oil production and stable production, and enhancing waterflooding recovery. In order to achieve "injection of good water" and "injection of enough water," petroleum engineers continued to carry out relevant research work. In this paper, an online suspended particle detector is proposed and developed for the downhole reinjection water quality testing. The light scattering method is used to realize the on-line detection of the content and median diameter of suspended particles in the reinjection water. The instrument includes laser, laser filter-collimated beam expander system, sample cell, Fourier transform lens system, detector and data processing system. Its core component is a combined multi-element silicon photodetector. In order to increase the detection area, the detector array adopts a semi-circular structure; that is, the structure of the detector array is composed of concentric half rings with different radii on one silicon wafer and independent of each other. Adjacent two rings are insulated from each other. A small hole is opened in the center of the detector array to pass the zero-order diffracted light.

On the basis of this physical hardware, this paper uses genetic algorithm and least squares algorithm to construct a contrast analysis of the anti-inference method of the online suspended particle detector. It can be seen that when the genetic algorithm is used for inversion calculation, the error is within $1 \%$ and the program running time is $8.35 \mathrm{~s}$. The algorithm has a high precision and a long operation time. When the least squares algorithm is used most for inversion calculation, the error is within $4 \%$ and the program running time is $0.22 \mathrm{~s}$. The algorithm has a short operation time and a poor precision. On the basis of reducing the requirement for accuracy, the least squares algorithm is more suitable for the online detection requirements of the system.

The on-line detection system of suspended particles in oilfield reinjection water finally adopts the least squares 
algorithm in this paper. However, in practical application, targeted non-negative least squares are designed to prevent negative results after the iteration. The simulation proves that it is feasible to use non-negative least-squares method for the on-line detection system of suspended particles in the oilfield reinjection water.

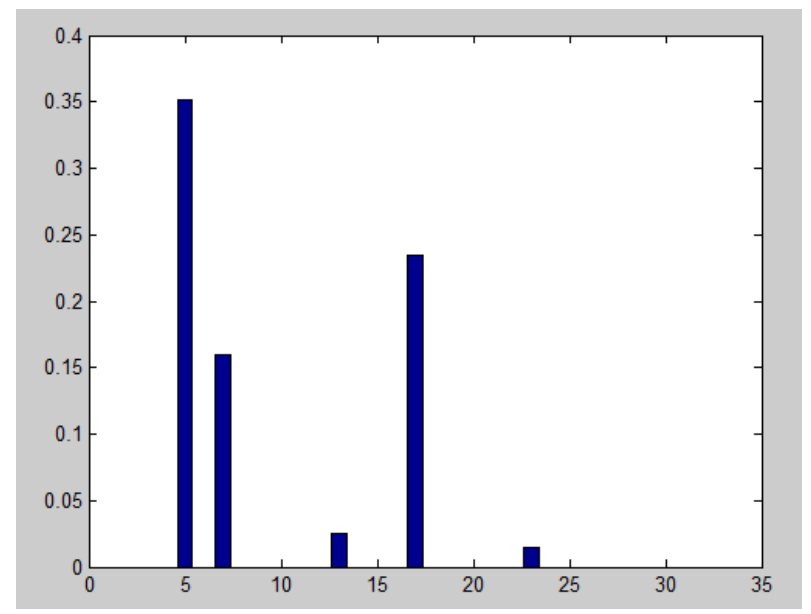

(a) Theoretical particle size distribution

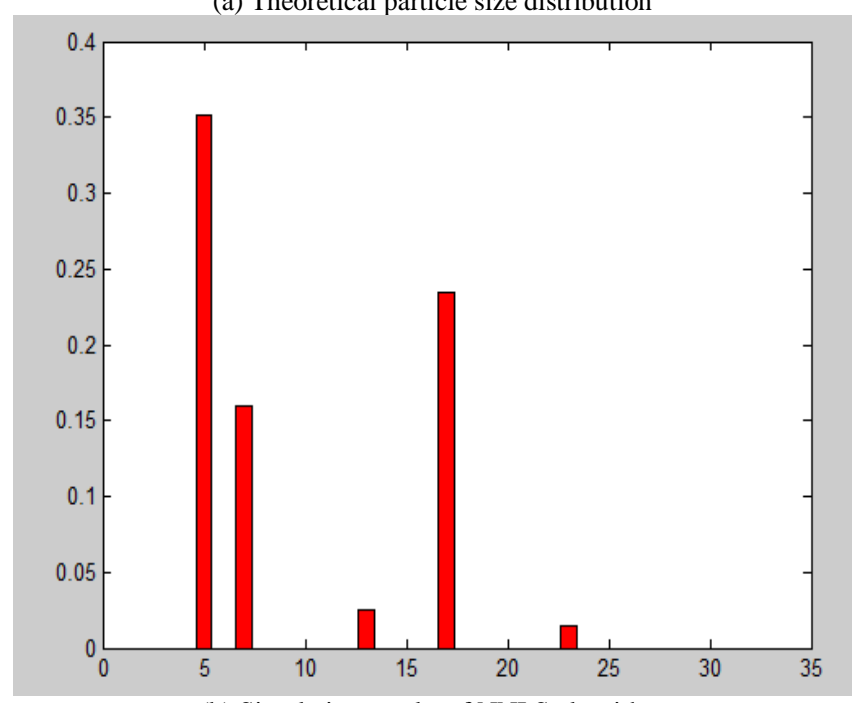

(b) Simulation results of NNLS algorithm

Figure 10. Multi-particle size distribution simulation results

\section{Acknowledgements}

This work is supported by Sino-us energy international cooperation project "energy and water bond and the key technology of efficient green utilization" (2017-40220-000001).

\section{References}

1. Gao Jianchong, Li Haitao, Qiao Wenbo, Shan Jincheng, Zhang Ling, "The Compatibility between Suspended Solids Partice Size and Pore Throat during Water-Flooding in Qikuo 17-2 Oilfield", Journal of Petrochemical Universities, vol.27, pp. 56-59, 89, 2014.

2. Hu Zeng, Chen Jian, Zhou Danong, Sun Jiyong, "Development of a Liquid Particle Counter based on Light Scattering Structuce", Analutical Instrumentation, No.5, pp.11-14, 2017.

3. Huang Qiyu, Bi Quan, “Application of New Technology for Wastewater Trertment in Low Permeabilitey oilfield”. Journal of Petrochemical Universities, vol.28, pp. 69-73, 2015.

4. Jia Deli, Pei Xiaohan, "Research on the Flow Control Strategy of Water Distributor in Water Injection Well", The 33rd Chinese Control Conference, pp. 4993-4996, 2014.

5. Jiang Wnalu, Lei Yafei, Dai Haodong, Ynag Chao, Zhang Sheng, "Hunidity Modified Light Scattering Method Applied in Field Dust Monitoring", Chinese Journal of Scientific Instrument, vol.39, pp.200-207, 2018.

6. Lin Y, Wang C, Ma C, et al: "A new combination method for multisensor conflict information," Journal of Supercomputing, Vol.72, No.7, pp. 2874-2890 2016. 
7. Lin Y, Zhu X, Zheng Z, et al. "The individual identification method of wireless device based on dimensionality reduction and machine learning”. Journal of Supercomputing, No.5, pp.1-18 2017.

8. Liu He, "High-efficiency measuring and adjusting technology and management for separated layer water injection". Beijing: Petroleum Industry Press, 2016.

9. Liu He, Pei Xiaohan, Jia Deli, Sun Fuchao, Guo Tong, "Connotation, application and prospect of the fourth-generation separated layer water injection technology”, Petroleum Exploration and Development, vol.44, pp. 608-614, 2017.

10. Liu He, Pei Xiaohan, Luo Kai, Sun Fuchao,Zheng Licheng, Yang Qinghai, "Current status and trend of separated layer water flooding in china". Petroleum Exploration and Development, vol.40, pp. 733-737, 2013.

11. Liu He, Pei Xiaohan, Jia Deli, et al, "Real time monitoring technique of injection distribution in layered water injection". Proceedings of the 14th annual conference of automation of petroleum and chemical industry of China, pp.531-534, 2015.

12. Ma Jinging, Jiang Xiaoping, Peng Lingshu, "Application of genetic algorithm in inversing particle size distribution", Computer Engineering and Design, vol.33, pp.1051-1054, 2011.

13. Shi C, Dou Z, Lin Y, et al. "Dynamic threshold-setting for RF-powered cognitive radio networks in non-Gaussian noise". Physical Communication, Vol. 27, No. 4, pp. 99-105, 2018.

14. Sun Fuchao, Pei Xiaohan, Jia Deli, et al, "Research and application of real time monitoring technology of high efficiency water injection matching". Proceedings of the 14th annual conference of automation of petroleum and chemical industry of China, pp.523-526, 2015.

15. Wang Qingquan, Li Xuyu, Zhang Maolin, "Different Turbidity Fast Detection Technology Based on CCD", Instrument Technique and Sensor, No.1, pp.97-101, January 2013.

16. Wang Wenjing, Liu Wei, Chen Wengang, John C, Thomas, Wang Yajing, Shen Jin, "The Conversion of Particle Size Distributions Based on Mie Theory", The Journal of Light Scattering, vol.30, pp. 6-9, 2018.

17. Wu Qi, "Matching technology for improving the effect of water injection". Beijing: China Petrochemical Press, 2010.

18. Wu Q, Li Y, Lin Y: "The application of nonlocal total variation in image denoising for mobile transmission," Multimedia Tools \& Applications, Vol.76, No.16, pp. 1-13 2016.

19. Xie Fengqiang, "Expermental Evaluation of Formation Damage Caused by Particles in the Injected Water". Journal of Petrochemical Universities, vol.29, pp. 43-46, 2016.

20. Xu Yishu, Liu Xiaowei, Cui Jiang, Chen Dong, Han Jinke, Xu Minghou, "Mass Concentration Measurements of the Coalderived Fiy Ash Particles Via Lsght Extinction Method", Journal of Engineering Thermophysics, vol.38, pp.1496-1502, 2017.

21. Xue Xiaokang, Li Xiaoyu, Ding Mao, "Spectrum Interpretation and Processing of Laser Raman Spectroscopy", Chinese Journal of Inorganic Analytical Chemistry, vol.8, pp.66-70, April 2018.

22. Yan Weipeng, Yang Tao, Li Xin, Huang Fuxi, Wu Xiaozhi, Tang Hui, "Geological Characteristics and Hydrocarbon Exploration Potential of Lacustrine Carbonate Rock in China”. China Petroleum Eeploration, vol.19,.pp. 11-17, 2014.

23. Yin Yonghui, Yan Xinping, Xiao Hanliang, Wang Chengtao, "On Optic-scattering Measurement of Particle Size", Journal of Wuhan University of Technology (Transportation Science \& Engineering ), vol.27, pp.643-645, 2003.

24. Zou Ruijie, Chen Yubang, Fang Yanjun, Zhao Youquan, "Research on online detection technology of mineral oil in water based on Mie scattering theory", Chinese Journal of Scientific Instrument, vol.33, pp.655-660, 2018.

25. Yun Lin, Chao Wang, Jiaxing Wang, Zheng Dou. "A Novel Dynamic Spectrum Access Framework Based on Reinforcement Learning for Cognitive Radio Sensor Networks”. Sensors, Vol.16,No.10,pp. 1-22 2016. 